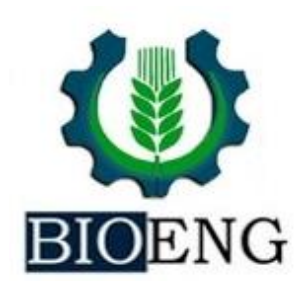

\title{
VISÃO ARTIFICIAL PARA A DIAGNOSE NUTRICIONAL DO MILHO CULTIVADO COM SILICATO DE CÁLCIO E MAGNÉSIO EM DOSES PONDERAIS E ALTAS DILUIÇÕES
}

\author{
M. M. Baesso ${ }^{1 *}$, T. M. G. Aprilanti' ${ }^{1}$, A. J. Modolo², F. Rossi ${ }^{1}$
}

${ }^{1}$ Faculdade de Zootecnia e Engenharia de Alimentos (FZEA) -

Universidade de São Paulo (USP), Pirassununga, São Paulo, Brasil.

${ }^{2}$ Universidade Tecnológica Federal do Paraná, Pato Branco - Paraná, Brasil.

Article history: Received 02 March 2020; Received in revised form 10 March 2020; Accepted 10 March 2020; Available online 31 March 2020.

\section{RESUMO}

A hipótese do presente trabalho foi que um sistema de visão artificial fosse capaz de caracterizar a deficiência nutricional na folha do milho, sob preparados homeopáticos, com a utilização das propriedades espectrais da cultura. O trabalho, realizado na Faculdade de Zootecnia e Engenharia de Alimentos (FZEA) - Universidade de São Paulo (USP), em Pirassununga/SP/Brasil estudou o comportamento nutricional da cultura do milho (Zea mays L.), hibrido Biogene 7049H. O delineamento experimental utilizado foi o blocos ao acaso com 6 tratamentos: silicato de cálcio e magnésio, nas seguintes dinamizações CH6 (diluição a 10-12), CH9 (diluição a $10^{-18}$ ), CH12 (diluição a $10^{-24}$ ) e $\mathrm{CH} 15$ (diluição a $10^{-30}$ ), um tratamento controle sem aplicação de silício e um tratamento com silicato de cálcio e magnésio na dose de $1 \mathrm{t} \mathrm{ha}^{-1}$, com 10 repetições. As folhas de milho foram digitalizadas por "scanner" e o processamento das imagens foi realizado pelo programa MATLAB ${ }^{\circledR}$. Três diferentes tamanhos de blocos de imagem foram testados 9 × 9; 20 x 20 e 40 x 40 "pixels". Os melhores resultados foram alcançados pelos blocos de 40 x 40 "pixels".

Palavras-chave: agricultura de precisão, processamento de imagens digitais, classificador estatístico, homeopatia.

\section{ARTIFICIAL VISION FOR NUTRITIONAL DIAGNOSIS OF CORN GROWN WITH CALCIUM SILICATE AND MAGNESIUM IN PONDERAL DOSES AND HIGH DILUTIONS}

\begin{abstract}
The hypothesis of the present work was that an artificial vision system was able to characterize the nutritional deficiency in the corn leaf, under homeopathic preparations, using the spectral properties of the culture. The work, carried out at the Faculty of Zootechnics and Food Engineering (FZEA) - University of São Paulo (USP), in Pirassununga / SP / Brazil studied the nutritional behavior of corn (Zeamays L.), hybrid Biogene $7049 \mathrm{H}$. The experimental design used was a randomized block with 6 treatments: calcium and magnesium silicate, in the following dynamizations $\mathrm{CH} 6$ (dilution at 10-12), $\mathrm{CH} 9$ (dilution at 10-18), CH12 (dilution at 10-24) and $\mathrm{CH} 15$ (dilution to 10-30), a control treatment without application of silicon and a treatment with calcium and magnesium silicate at a dose of $1 \mathrm{t}$ ha1 , with 10 repetitions. The corn leaves were digitized by "scanner" and the image processing was performed by the MATLAB $®$ program. Three different sizes of image blocks were tested $9 \times 9 ; 20 \times 20$ and $40 \times 40$ "pixels". The best results were achieved by the blocks of $40 \times 40$ "pixels".
\end{abstract}

Keywords: precision agriculture, digital image processing, statistical classifier, homeopathy.

baesso@usp.br 


\section{INTRODUÇÃO}

Considerado um dos principais alimentos da nutrição humana e animal em todo o mundo, o milho tem desempenhado papel essencial na economia familiar brasileira e no agronegócio como um todo. De acordo com perspectivas do governo brasileiro para a safra de 2018/2019, é esperado um recorde de produção com 91,1 milhões de toneladas, produzidas em 16,67 milhões de hectares (CONAB, 2018).

O aumento de disponibilidade de silicato de cálcio e magnésio para as plantas tem melhorado o desenvolvimento e aumentado a produtividade de diversas culturas (MIRANDA et., 2018). O silício (Si) é o segundo mineral mais abundante na crosta terrestre, porém sua disponibilidade solúvel no solo é variável em função dos minerais e textura. Tipos de solo com menor quantidade de minerais primários e secundário e consequentemente, maior teor de óxidos de ferro e alumínio, tendem a ter menor teor de Si no solo.

A forma de fornecimento de Si para as plantas vem sendo estudada a partir da liberação do uso do silicato como fertilizante. Os silicatos são aplicados principalmente na forma sólida (pó ou granulado), mas também podem ser aplicados na forma líquida (via solo ou via foliar). A forma de diluição para aplicação do silicato de cálcio e magnésio pode ser feita com base a obter doses homeopáticas da solução.

Uma maneira prática e rápida de determinar o estado nutricional da planta é por meio do diagnóstico visual. Porém, a

\section{MATERIAL E MÉTODOS}

O trabalho foi realizado na Faculdade de Zootecnia e Engenharia de Alimentos (FZEA) - Universidade de São Paulo (USP), em Pirassununga/SP/Brasil. A cultura estudada foi o milho (Zea mays L.), hibrido Biogene 7049H, conduzido em sua precisão está limitada à experiência do técnico e, também, exige bastante trabalho para gerar um mapa de prescrição para ser utilizado no manejo localizado (ROMUALDO et al., 2018). Em aplicações convencionais de fertilizantes, toda a área de um talhão é considerada homogênea, ou seja, a necessidade de adubo é tida como igual para toda a área, o que na realidade não ocorre. Baseando-se nas necessidades médias da planta, uma aplicação uniforme causa adubação excessiva em algumas áreas do campo e insuficiente em outras, com comprometimento da eficiência do processo de produção. Nesses casos, a agricultura de precisão é uma alternativa ao sistema convencional, com aplicação de nutrientes em doses variadas (ROMUALDO et al., 2014).

Um sistema de visão artificial é aquele capaz de obter, processar e interpretar imagens. $\mathrm{O}$ objetivo do desenvolvimento destes sistemas é tentar imitar o raciocínio humano, em qualquer campo onde se faça necessário $\mathrm{o}$ reconhecimento de padrões visuais. $\mathrm{Na}$ área agrícola, este tipo de sistema tem sido usado em aplicações localizadas de adubos, herbicidas, inseticidas e fungicidas, entre outros.

Com a finalidade de explorar o potencial dessa tecnologia na agricultura, a hipótese do presente trabalho foi que um sistema de visão artificial fosse capaz de caracterizar a deficiência nutricional na folha do milho, com a utilização das propriedades espectrais da cultura.

casa de vegetação. As parcelas experimentais foram constituídas por vasos de $5 \mathrm{dm}^{3}$, preenchidos com $3 \mathrm{~kg}$ de uma mistura de solo (Tabela 1), areia e esterco bovino curtido, na proporção de 2:1:1, respectivamente, nos quais foram 
conduzidas três plantas de milho. Durante a condução do experimento o ambiente protegido apresentou temperatura máxima de $34,7^{\circ} \mathrm{C}$ e mínima $20,4^{\circ} \mathrm{C}$, e umidade relativa do ar de $70,4 \%$ máxima e $28,3 \%$ mínima.

Tabela 1. Análise química do solo do experimento

\begin{tabular}{cccccccccccc}
\hline $\begin{array}{c}\mathbf{p H} \\
\mathbf{C a C l}\end{array}$ & $\begin{array}{c}\mathbf{M . O} \\
\mathbf{g} / \mathbf{k g}\end{array}$ & $\begin{array}{c}\mathbf{P} \\
\mathbf{m g ~ d m}^{-3}\end{array}$ & $\mathbf{S}$ & $\mathbf{K}$ & $\mathbf{C a}$ & $\mathbf{M g}$ & $\begin{array}{c}\mathbf{A l} \\
\mathbf{m m o l c}^{2}\end{array}$ & $\begin{array}{c}\mathbf{H + A l} \\
\mathbf{d m}^{-3}\end{array}$ & $\mathbf{S B}$ & $\mathbf{C T C}$ & $\begin{array}{c}\mathbf{V} \\
\mathbf{\%}\end{array}$ \\
\hline 5,6 & 10 & 15 & 5 & 1,2 & 21 & 7 & 0 & 15 & 0,40 & 44 & 66 \\
\hline
\end{tabular}

\begin{tabular}{ccccc}
\hline B & $\mathbf{C u}$ & $\begin{array}{c}\mathbf{F e} \\
\mathbf{m g ~ d m}^{-3}\end{array}$ & Mn & $\mathbf{Z n}$ \\
\hline 0,40 & 0,6 & 27 & 3 & 0,4 \\
\hline
\end{tabular}

O delineamento experimental utilizado foi blocos ao acaso com 6 tratamentos: silicato de cálcio e magnésio, preparado segundo as normas da farmacopeia homeopática brasileira (ANVISA, 2011), nas seguintes dinamizações $6 \mathrm{CH}$ (diluição a $10^{-12}$ ), $9 \mathrm{CH}$ (diluição a $10^{-18}$ ), 12CH (diluição a $10^{-24}$ ) e $15 \mathrm{CH}$ (diluição a $10^{-30}$ ), um tratamento controle sem aplicação de silicato de cálcio e magnésio e um tratamento com silicato de cálcio e magnésio na dose de $1 \mathrm{t} \mathrm{ha}^{-1}$, adicionado previamente ao solo, com 10 repetições.

Os tratamentos foram aplicados em sistema de duplo-cego, ou seja, os tratamentos dinamizados foram identificados por números, sendo desconhecidos do aplicador. As dinamizações de aplicação foram preparadas diariamente em água deionizada, sendo que o tratamento controle, sem aplicação de cálcio e magnésio, foi preparado a partir da solução alcoólica $70 \%$ (utilizado como diluente nos demais tratamentos). A aplicação dos tratamentos ocorreu a partir de 3 dias após a emergência (DAE) do milho, aplicandose 5 gotas $(0,2 \mathrm{~mL})$ por vaso, diariamente.

O solo dos vasos foi mantido na umidade da capacidade de campo, não havendo desse modo deficiência hídrica para o desenvolvimento das plantas.
Para a obtenção das imagens, após 15 dias da aplicação dos tratamentos às plantas do milho (fase vegetativa com 5 folhas-V5), foram coletadas as folhas indicativas de duas plantas de cada vaso da parcela, totalizando quatro folhas por tratamento. As folhas foram cortadas na base e em seguida digitalizadas com resolução de 1200 dpi utilizando um scanner convencional de mesa, as imagens foram armazenadas no formato TIFF ("tagged image file format").

O processamento das imagens foi realizado no Laboratório de Mecanização Agrícola e Agricultura de Precisão do Departamento de Engenharia de Biossistemas (LAMAP) da Faculdade de Zootecnia e Engenharia de Alimentos. Foi utilizado o sistema computacional MATLAB $^{\circledR}$ com o pacote de ferramentas para imagens.

No processamento foi retirada características das imagens, condensando as informações nelas contidas. Dessa maneira, em vez de se utilizar a imagem original, utilizou-se um vetor com características representativas da informação desejada. Este trabalho partiu da hipótese que quatro índices espectrais levam a informação desejada para classificar os tratamentos de acordo com o nível de nutrientes determinados na análise química foliar.

As características calculadas foram: 


$$
\begin{gathered}
E V d=\frac{2 \cdot V d-V m-A z}{V m+V d+A z} \\
V e r n=\frac{V m}{V m+V d+A z} \\
V n=\frac{V d}{V m+V d+A z} \\
R v v=\frac{V d}{V m}
\end{gathered}
$$

em que,

Evd = excesso de verde;

$\mathrm{Vd} \quad=$ média dos pixels na banda do verde; $\mathrm{V}$

Vern = vermelho normalizado;

$\mathrm{Az} \quad=$ média dos pixels na banda do azul; $\quad \mathrm{Rvv} \quad$ = razão verde-vermelho.

$\mathrm{Vm} \quad=$ média dos pixels na banda do vermelho;

$\mathrm{O}$ índice excesso de verde $(\mathrm{EVd})$ vem sendo utilizado principalmente para facilitar a discriminação do material verde (vegetal) em relação ao solo. No presente trabalho, partiu-se da hipótese de que o realce da informação na banda verde poderia melhorar o poder de discriminação entre os tratamentos. Também se testou a utilização de apenas uma banda ( $\mathrm{Vm}$ e Vern), possibilitando futuramente a utilização de câmera monocromática com filtro óptico. Por último, utilizou-se a combinação das duas bandas de maior relação com as propriedades espectrais das plantas no visível (Rvv).

Essas características foram calculadas de sub-blocos da imagem que representava a refletância da folha. Foram testados sub-blocos da imagem com dimensões de 9x9, 20x20 e 40x40 pixels. Cada parcela teve cinco sub-blocos, sendo que a média dos valores de pixels desses sub-blocos representou uma observação do vetor de característica. Para recortar os sub-blocos, foi desenvolvido um programa computacional implementado no MATLAB $^{\circledR}$, no qual as imagens foram cortadas no tamanho escolhido e em regiões aleatórias.

$\mathrm{O}$ vetor de características foi utilizado como variável de entrada de um classificador para discriminar as doses de silício. Todas as 15 combinações de índices possíveis foram testadas (Tabela 2).

Tabela 2. Vetores de características (X) que foram testados no desenvolvimento dos classificadores para discriminar diferentes níveis de silício na folha de milho.

\begin{tabular}{l|l|l|l}
\hline \multicolumn{1}{c|}{ Um índice } & \multicolumn{1}{|c|}{ Dois índices } & \multicolumn{1}{c}{ Três índices } & \multicolumn{1}{c}{ Quatro índices } \\
\hline $\mathrm{X}=$ Evd & $\mathrm{X}=$ Evd e Vern & $\mathrm{X}=$ Evd, Vern e Vn & $\begin{array}{l}\mathrm{X}=\text { Evd, Vern, Vn e } \\
\text { Rvv }\end{array}$ \\
$\mathrm{X}=$ Vern & $\mathrm{X}=$ Evd e Vn & $\mathrm{X}=$ Evd, Vern e Rvv & \\
$\mathrm{X}=$ Vn & $\mathrm{X}=$ Evd e Rvv & $\mathrm{X}=$ Evd, Vn e Rvv & \\
$\mathrm{X}=\mathrm{Rvv}$ & $\mathrm{X}=$ Vern e Vn & $\mathrm{X}=$ Vern, Vn e Rvv & \\
& $\mathrm{X}=$ Vern e Rvv & & \\
& $\mathrm{X}=$ Rvv e Vn & & \\
\hline
\end{tabular}

Para a análise das imagens foi testado um classificador estatístico, descrito por Gonzalez \& Woods (1992), para avaliar o status nutricional do milho. 
O classificador estatístico foi composto por

um conjunto de funções discriminantes:

$$
d_{j}(X)=\ln P\left(W_{j}\right)-\frac{1}{2} \ln \left(\operatorname{det} C_{j}\right)-\frac{1}{2}\left[\left(X-m_{j}\right)^{T} C_{j}^{-1}\left(X-m_{j}\right)\right]
$$

em que,

$\mathrm{d}_{j}=$ função discriminante da classe $\mathrm{j}$;

$\mathrm{C}_{j}=$ matriz de covariância da classe j;

det $\mathrm{C}_{j}=$ determinante da matriz de covariância da classe $\mathrm{j}$;

$\mathrm{X}=$ vetor de características;

$\mathrm{m}_{j}=$ vetor de médias da classe $\mathbf{j}$; característica;

$\mathrm{n}=$ dimensão do vetor de $\mathrm{P}\left(\mathrm{W}_{j}\right)=$ probabilidade a priori da classe $\mathrm{j}$; e

$\mathrm{T}=$ símbolo que representa a transposta de uma matriz.

Foi considerada a mesma probabilidade, a priori, de ocorrência dos tratamentos. Desta maneira, a equação 5 foi escrita como:

$$
d_{j}(X)=-\frac{1}{2} \ln (\operatorname{det} C j)-\frac{1}{2}\left[\left(X-m_{j}\right)^{T} C_{j}^{-1}\left(X-m_{j}\right)\right]
$$

Seis funções discriminantes foram desenvolvidas, uma para cada tratamento estudado, de forma que um vetor desconhecido $\mathrm{X}$ foi atribuído à classe $\mathrm{j}$, que apresentou o maior valor da função discriminante $\mathrm{d}_{j}(\mathrm{X})$.

O classificador estatístico também foi utilizado para definição do melhor vetor de características. $O$ erro de classificação foi estimado por validação cruzada deixando um de fora (KHATTREE \& NAIK, 2000). Uma observação foi separada e as funções discriminantes foram elaboradas com os restantes dos dados (59 amostras). Em

$$
E G=\frac{C C}{n_{t}} x 100
$$

em que,

$\mathrm{EG}=$ exatidão global;

$\mathrm{CC}=$ número de amostras classificadas corretamente; e seguida, a observação separada foi classificada. Esse procedimento foi repetido sucessivamente para cada amostra, ou seja, todas as 60 observações (6 tratamentos, 10 repetições), de cada dimensão de bloco, foram testadas. $\mathrm{O}$ erro de classificação foi estimado pela elaboração de uma matriz de erro, conforme descrito em Congalton (1991).

A mensuração do desempenho dos classificadores foi determinada pela exatidão global de classificação e pelo coeficiente Kappa.

A exatidão global do classificador foi estimada pela seguinte equação:

$\mathrm{N}_{t}=$ número total de amostras.

O coeficiente Kappa foi estimado pela seguinte equação: 


$$
\hat{K}=\frac{n_{t} \sum_{i=1}^{c} x_{i i}-\sum_{i=1}^{c} x_{i \oplus} x_{\oplus i}}{n_{t}^{2}-\sum_{i=1}^{c} x_{i \oplus} x_{\oplus i}}
$$

em que,

$\hat{K}=$ Estimativa do coeficiente Kappa;

Por fim, para definir os melhores

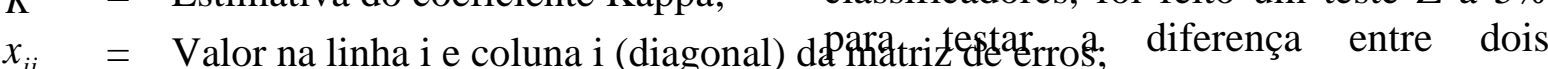

$x_{i \oplus}=$ total da linha i (erro de inclusão);

$x_{\oplus i}=$ total da coluna $\mathrm{i}$ (erro de omissão); $\mathrm{e}$

$\mathrm{C}=$ Número total de doses. classificadores.

$\mathrm{O}$ valor do $\mathrm{Z}$ calculado foi determinado pela seguinte equação:

$$
Z=\frac{\hat{K}_{1}-\hat{K}_{2}}{\sqrt{\operatorname{var}\left(\hat{K}_{1}\right)+\operatorname{var}\left(\hat{K}_{2}\right)}}
$$

em que,

$$
\begin{aligned}
Z & =\text { Valor de Z calculado; } \\
\hat{K}_{1} & =\text { Coeficiente Kappa1; } \\
\hat{K}_{2} & =\text { Coeficiente Kappa2; }
\end{aligned}
$$

$\operatorname{var}\left(\hat{K}_{1}\right)=$ Variância do coeficiente Kappa1; e $\operatorname{var}\left(\hat{K}_{2}\right)=$ Variância do coeficiente Kappa2.

A variância do coeficiente Kappa foi determinada pelas equações 10 a 14 .

$$
\operatorname{var}(\hat{K})=\frac{1}{n_{t}}\left[\frac{\theta_{1}\left(1-\theta_{1}\right)}{\left(1-\theta_{2}\right)^{2}}+\frac{2\left(1-\theta_{1}\right)\left(2 \theta_{1} \theta_{2}-\theta_{3}\right)}{\left(1-\theta_{2}\right)^{3}}+\frac{\left(1-\theta_{1}\right)^{2}\left(\theta_{4}-4 \theta_{2}^{2}\right)}{\left(1-\theta_{2}\right)^{4}}\right]
$$

em que,

$$
\begin{aligned}
& \theta_{1}=\frac{1}{n_{t}} \sum_{i=1}^{c} x_{i i} \\
& \theta_{2}=\frac{1}{n_{t}^{2}} \sum_{i=1}^{c} x_{i \oplus} x_{\oplus i} \\
& \theta_{3}=\frac{1}{n_{t}^{2}} \sum_{i=1}^{c} x_{i i}\left(x_{i \oplus}+x_{\oplus i}\right) \\
& \theta_{4}=\frac{1}{n_{t}^{3}} \sum_{i=1}^{c} \sum_{j=1}^{c} x_{i j}\left(x_{j \oplus}+x_{\oplus i}\right)^{2}
\end{aligned}
$$

Também foram realizadas análises químicas das folhas amostradas e classificadas. Para esse procedimento todo o material foi lavado e seco em estufa de circulação forçada de ar à temperatura de $65^{\circ} \mathrm{C}$ por 72 horas. As amostras foram moídas e acondicionadas em sacos plásticos, para posterior determinação do 
teor foliar dos nutrientes. As análises foram realizadas no Laboratório de Solos e Plantas do Departamento de Zootecnia da Faculdade de Zootecnia e Engenharia de Alimentos (FZEA-USP). Os resultados

\section{RESULTADOS E DISCUSSÕES}

Utilizando os critérios sugeridos por Romualdo et al. (2018), para os blocos de $9 \times 9$, seis casos tiveram classificação ruim e foram submetidos a análise de variância e, nos casos em que $o$ teste $F$ foi significativo, foi realizada teste ScottKnott para comparação das médias a $5 \%$ de probabilidade.

os outros nove casos não diferem estaticamente de zero (Tabela 3).

Tabela 3. Exatidão global e coeficiente Kappa das funções discriminantes, utilizando blocos de imagens de 9x9 pixels, para cada combinação de índices testados.

\begin{tabular}{c|c|c}
\hline Índices Espectrais & Exatidão Global & Kappa \\
\hline Evd & 0,36 & $0,20^{\mathrm{a}}$ \\
Vn & 0,36 & $0,20^{\mathrm{a}}$ \\
Rvv & 0,34 & $0,17^{\mathrm{a}}$ \\
Evd e Rvv & 0,32 & $0,15^{\mathrm{a}}$ \\
Vn e Rvv & 0,32 & $0,15^{\mathrm{a}}$ \\
Vern e Rvv & 0,32 & $0,15^{\mathrm{a}}$ \\
Evd e Vern & 0,30 & $0,12^{\mathrm{ns}}$ \\
Vern e Vn & 0,30 & $0,12^{\mathrm{ns}}$ \\
Evd, Vern e Rvv & 0,28 & $0,10^{\mathrm{ns}}$ \\
Vern, Vn e Rvv & 0,28 & $0,10^{\mathrm{ns}}$ \\
Evd, Vern, Vn e Rvv & 0,28 & $0,10^{\mathrm{ns}}$ \\
Vern & 0,28 & $0,10^{\mathrm{ns}}$ \\
Evd, Vern e Vn & 0,26 & $0,07^{\mathrm{ns}}$ \\
Evd, Vn e Rvv & 0,26 & $0,07^{\mathrm{ns}}$ \\
Evd e Vn & 0,22 & $0,02^{\mathrm{ns}}$ \\
\hline
\end{tabular}

$\mathrm{ns}=$ não diferem de zero pelo teste $\mathrm{Z}$ a $5 \%$ de probabilidade; Os coeficientes Kappa seguidos da mesma letra não diferem significativamente pelo teste $\mathrm{Z}$ a $5 \%$ de probabilidade.

Os blocos de 20x20 pixels apresentaram classificação razoável para duas combinações de índices, oito casos são classificados como ruins e cinco casos não diferem estaticamente de zero (teste $\mathrm{Z}$ a 5\%) (Tabela 4). 
Tabela 4. Exatidão global e coeficiente Kappa das funções discriminantes, utilizando blocos de imagens de 20x20 pixels, para cada combinação de índices testados.

\begin{tabular}{c|c|c}
\hline Índices Espectrais & Exatidão Global & Kappa \\
\hline Evd, Vn e Rvv & 0,40 & $0,25^{\mathrm{a}}$ \\
Evd, Vern e Vn & 0,40 & $0,25^{\mathrm{a}}$ \\
Vern, Vn e Rvv & 0,36 & $0,20^{\mathrm{a}}$ \\
Vern e Rvv & 0,36 & $0,20^{\mathrm{a}}$ \\
Vn e Rvv & 0,36 & $0,20^{\mathrm{a}}$ \\
Evd e Rvv & 0,36 & $0,20^{\mathrm{a}}$ \\
Evd e Vern & 0,34 & $0,17^{\mathrm{a}}$ \\
Vern e Vn & 0,34 & $0,17^{\mathrm{a}}$ \\
Evd, Vern e Rvv & 0,34 & $0,17^{\mathrm{a}}$ \\
Evd, Vern, Vn e Rvv & 0,32 & $0,15^{\mathrm{a}}$ \\
Rvv & 0,30 & $0,12^{\mathrm{ns}}$ \\
Vn & 0,30 & $0,12^{\mathrm{ns}}$ \\
Evd & 0,30 & $0,12^{\mathrm{ns}}$ \\
Vern & 0,26 & $0,07^{\mathrm{ns}}$ \\
Evd e Vn & 0,26 & $0,07^{\mathrm{ns}}$ \\
\hline
\end{tabular}

ns= não diferem de zero pelo teste $\mathrm{Z}$ a $5 \%$ de probabilidade; Os coeficientes Kappa seguidos da mesma letra não diferem significativamente pelo teste $\mathrm{Z}$ a $5 \%$ de probabilidade.

Para os blocos de 40x40 pixels, seguindo o mesmo critério, a classificação foi considerada boa em três casos, razoável em oito, ruim em dois e não difere estaticamente pelo teste $\mathrm{Z}$ a $5 \%$ em dois casos (Tabela 5). Observa-se também que os melhores desempenhos ocorreram com a combinação de índices, indicando que a utilização de apenas um índice pode comprometer o desempenho da discriminação dos diferentes tratamentos utilizado neste trabalho. Resultados semelhantes foram encontrados por vários autores em diferentes culturas (LE REST et al., 2014). 
Tabela 5. Exatidão global e coeficiente Kappa das funções discriminantes, utilizando blocos de imagens de 40x40 pixels, para cada combinação de índices testados.

\begin{tabular}{c|c|c}
\hline Índices Espectrais & Exatidão Global & Kappa \\
\hline Evd,Vern e Vn & 0,66 & $0,57^{\mathrm{a}}$ \\
Evd e Vern & 0,54 & $0,42^{\mathrm{ab}}$ \\
Vern e Vn & 0,54 & $0,42^{\mathrm{ab}}$ \\
Vn e Rvv & 0,48 & $0,35^{\mathrm{b}}$ \\
Vern e Rvv & 0,48 & $0,35^{\mathrm{b}}$ \\
Evd e Rvv & 0,46 & $0,32^{\mathrm{bc}}$ \\
Evd, Vn e Rvv & 0,42 & $0,27^{\mathrm{bc}}$ \\
Evd,Vern, Vn e Rvv & 0,42 & $0,27^{\mathrm{bc}}$ \\
Vern & 0,42 & $0,27^{\mathrm{bc}}$ \\
Evd,Vern e Rvv & 0,42 & $0,27^{\mathrm{bc}}$ \\
Rvv & 0,42 & $0,27^{\mathrm{bc}}$ \\
Vern, Vn e Rvv & 0,36 & $0,20^{\mathrm{c}}$ \\
Vn & 0,32 & $0,15^{\mathrm{c}}$ \\
Evd & 0,26 & $0,07^{\mathrm{ns}}$ \\
Evd e Vn & 0,26 & $0,07^{\mathrm{ns}}$ \\
\hline
\end{tabular}

$\mathrm{ns}=$ não diferem de zero pelo teste $\mathrm{Z}$ a $5 \%$ de probabilidade; Os coeficientes Kappa seguidos da mesma letra não diferem significativamente pelo teste $\mathrm{Z}$ a $5 \%$ de probabilidade.

Dessa maneira, apesar da sobreposição da sensibilidade nas diferentes bandas, devido à baixa resolução espectral da imagem, a utilização de mais de um índice espectral facilitou a discriminação dos diferentes tratamentos utilizados neste trabalho. A utilização de sensores com faixas de sobreposição menores, ou seja, com sensibilidade concentrada nas bandas de interesse (550 $\mathrm{nm}$ e $650 \mathrm{~nm}$ ), pode realçar as propriedades espectrais das plantas, melhorando a discriminação.

Os blocos 40x40 pixels foram os que proporcionaram melhores resultados. Dessa maneira, os mesmos podem ser recomendados para a utilização em um sistema de discriminação das imagens estudadas. Na Tabela 6 é apresentado o resultado da matriz de erro da melhor combinação de índices (Evd, Vern e Vn). 
Tabela 6. Matriz de erro obtida com o uso dos índices da melhor combinação de índices (Evd, Vern e Vn) para os blocos de 40x40 pixels

\begin{tabular}{c|cccccc|c}
\hline \multirow{2}{*}{$\begin{array}{c}\text { Valor } \\
\text { Estimado }\end{array}$} & \multicolumn{5}{|c|}{ Tratamento } & $\begin{array}{c}\text { Erros de } \\
\text { inclusão (\%) }\end{array}$ \\
\cline { 2 - 6 } & $1 \mathrm{t} \mathrm{ha}^{-1}$ & $\mathrm{CH} 6$ & $\mathrm{CH} 9$ & $\mathrm{CH} 12$ & $\mathrm{CH} 15$ & $\mathrm{H}_{2} \mathrm{O}$ & \\
\hline $1 \mathrm{t} \mathrm{ha}^{-1}$ & $\mathbf{6}$ & 3 & 0 & 0 & 0 & 0 & 33 \\
$\mathrm{CH} 6$ & 4 & $\mathbf{7}$ & 0 & 0 & 0 & 0 & 36 \\
$\mathrm{CH} 9$ & 0 & 0 & $\mathbf{6}$ & 1 & 2 & 2 & 45 \\
$\mathrm{CH} 12$ & 0 & 0 & 1 & $\mathbf{7}$ & 1 & 2 & 36 \\
$\mathrm{CH} 15$ & 0 & 0 & 2 & 0 & $\mathbf{5}$ & 0 & 29 \\
$\mathrm{H} 2 \mathrm{O}$ & 0 & 0 & 1 & 2 & 2 & $\mathbf{6}$ & 45 \\
\hline $\begin{array}{c}\text { Erros de } \\
\text { omissão (\%) }\end{array}$ & 40 & 30 & 40 & 30 & 50 & 40 & $\begin{array}{c}\text { Exatidão } \\
\text { global= 62\% }\end{array}$ \\
\hline
\end{tabular}

Os acertos foram maiores que os erros na maioria dos tratamentos, o classificador acertou $62 \%$ das amostras. O erro de classificação faz com que um sistema de aplicação a taxa variada coloque mais ou menos adubo que o recomendado.

Destaca-se também o fato que os tratamentos CH6 (diluição a $10^{-12}$ ) e silicato de cálcio e magnésio na dose de $1 \mathrm{t}$ ha $^{-1}$, obtiveram erros de inclusão somente entre si, ou seja, não se confundiram com nenhum dos outros tratamentos, demostrando que obtiveram resultados semelhantes (Tabela 6). É importante ressaltar que a utilização de doses mínimas é uma forma de diminuir a toxicidade de substâncias em doses ponderais, conseguindo efeitos iguais ou superiores ao obtidos com doses massivas. É por isso que a homeopatia permite utilizar os princípios curativos de substâncias muito venenosas. Os demais tratamentos estudados CH9 (diluição a $10^{-18}$ ), CH12 (diluição a $10^{-24}$ ) e $\mathrm{CH} 15$ (diluição a $10^{-30}$ ), e um tratamento controle sem aplicação de silício, seguiram na mesma tendência, ou seja, não diferiam estatisticamente entre si, mostrando que essas diluições comprometeram a eficácia do tratamento, já que não obtiveram valores diferentes do tratamento com água. A Tabela 7 apresenta a análise foliar do milho com aplicação de silicato de cálcio e magnésio em doses ponderais e altas diluições. 
Tabela 7. Teores de cálcio (Ca), magnésio $(\mathrm{Mg})$ e silício $(\mathrm{Si})$ da parte aérea do milho, fase V5, com aplicação de silicato de cálcio e magnésio em doses ponderais e altas diluições

\begin{tabular}{lccc}
\hline Tratamentos & Ca & Mg & Si \\
\hline 1 t ha $^{-1}$ & $2,19 \mathrm{a}$ & $0,68 \mathrm{a}$ & $11335 \mathrm{a}$ \\
$\mathrm{CH} 6 *$ & $2,33 \mathrm{a}$ & $0,69 \mathrm{a}$ & $11791 \mathrm{a}$ \\
$\mathrm{CH} 9$ & $1,64 \mathrm{c}$ & $0,56 \mathrm{~b}$ & $9179 \mathrm{~b}$ \\
$\mathrm{CH} 12$ & $1,57 \mathrm{c}$ & $0,62 \mathrm{~b}$ & $8723 \mathrm{~b}$ \\
CH15 & $1,90 \mathrm{~b}$ & $0,63 \mathrm{~b}$ & $9303 \mathrm{~b}$ \\
Testemunha** & $1,68 \mathrm{c}$ & $0,59 \mathrm{~b}$ & $8350 \mathrm{~b}$ \\
\hline C.V. $(\%)$ & 10,36 & 8,90 & 22,53
\end{tabular}

Médias seguidas de letras distintas diferem entre si pelo teste de Scott-Knott a 5\% de probabilidade. *Silicato de cálcio e magnésio preparado de acordo com as normas da farmacopeia homeopática brasileira, na escala centesimal hahnemanniana (CH) ** Testemunha: álcool 70\%, diluído 1 parte em 99 partes de água deionizada.

Em relação ao nitrogênio $(\mathrm{N})$, fósforo $(\mathrm{P})$, potássio $(\mathrm{K})$ e enxofre $(\mathrm{S})$ não houve diferenças significativas entre os tratamentos, apresentando em média $11,13 \mathrm{~g} \mathrm{~kg}^{-1}, 3,16 \mathrm{~g} \mathrm{~kg}^{-1}, 33,75 \mathrm{~g} \mathrm{~kg}^{-1} \mathrm{e}$ $1,40 \mathrm{~g} \mathrm{~kg}^{-1}$, respectivamente para $\mathrm{N}, \mathrm{P}, \mathrm{K} \mathrm{e}$ S.

As análises foliares demostram que não houve diferença significativa para os teores de cálcio, magnésio e silício entre os tratamentos CH6 e silicato de cálcio e magnésio na dose $1 \mathrm{t} \mathrm{ha}^{-1}$. Deste modo, o resultado obtido pelo classificador fica validado.

\section{CONCLUSÕES}

Os melhores desempenhos de classificação ocorreram com a utilização de mais de um índice espectral no vetor de características do classificador.

Não houve diferença significativa para os teores foliares de cálcio e magnésio
$\mathrm{O}$ uso das funções discriminantes revelou baixa disponibilidade de nutrientes na folha, justificando a boa classificação realizada pelo algoritmo. Todavia é necessário que sejam realizadas pesquisas adicionais para verificar a variação do teor dos nutrientes ao longo do ciclo da cultura, principalmente dentro das doses recomendadas no campo, visando estimar as doses a serem aplicadas para corrigir possíveis deficiências e fornecer à cultura, o nutriente em dose e época adequadas.

e silício entre os tratamentos $\mathrm{CH} 6$ (diluição a $10^{-12}$ ) e silicato de cálcio e magnésio na dose de $1 \mathrm{t} \mathrm{ha}^{-1}$.

$\mathrm{O}$ uso das funções discriminantes revelou baixa disponibilidade de nutrientes na folha. 


\section{REFERÊNCIAS}

ANVISA - Agencia Nacional de Vigilância Sanitária:

FARMACOPEIA

Homeopática brasileira. Brasília. 364p, 2011.

CONAB - Companhia Nacional de Abastecimento: Acompanhamento da Safra Brasileira de Grãos. Brasilia, Conab, 127p, 2018.

CONGALTON, R. G.; A review of assessing the accuracy of classifications of remotely sensed data. Remote sensing thematic accuracy assessment, v. 37 (1): 35-46, 1991.

GONZALEZ, R. C.; WOODS, R. E.; (1992) Digital image processing. Massachusetts, Addison-Wesley Publishing Company. 716p, 1992.

KHATTREE, R.; MAIK, D. N.; Multivariate data reduction and discrimination with SAS software. Cary, SAS Institute Inc. 584p, 2000.

LE REST K.; PINAUD D.; MONESTIEZ P.; CHADOEUF J.; B.; Spatial leave-oneout cross-validation forvariable selection in the presence ofspatial autocorrelation. Global Ecology and Biogeography, v. 23 (7): 811-820, 2014.

MIRANDA, P.S.; MORAES, T. R.; SANTOS, J. R. E.; CARVALHO, F. D.; VIANA, J. P.; PÉREZ-MALUF, R.; Aplicação de silício na cultura do milho. Revista Ciência Agroambiental, v. 16 (1): 2-6, 2018.

ROMUALDO, L. M.; LUZ, P. H. C.; BAESSO, M. M.; DEVECHIO, F. F. S.; BET, J. A.; Spectral indexes for identification of nitrogen deficiency in maize. Revista Ciência Agronômica, v. 49 (2): 183-191, 2018.
ROMUALDO, L. M.; LUZ, P. H. C.; DEVECHIO, F. F.S.; MARIN, M. A.; ZÚNIGA, A. M. G.; BRUNO, O. M.; HERLING, V. R.; Use of artificial vision techniques for diagnostic of nitrogen nutritional status in maize plants. Computers and Electronics in Agriculture, v. 104 (2): 63-70, 2014. 